\title{
STUDIES ON PHOSPHATASES AND LIPASES IN CERTAIN TURBELLARIA
}

\author{
T: \\ PATH I. OGORKE
}

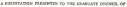

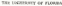

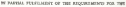

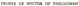

ioimaser ar poters

hept lass 


\section{Lavanwerames}

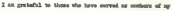

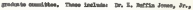

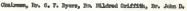

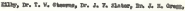

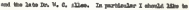

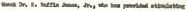

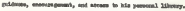

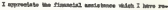

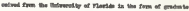

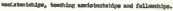

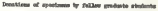

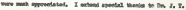

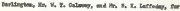

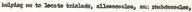
nepratenaly.

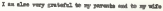

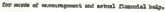


theit or cowre

na

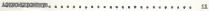

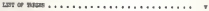

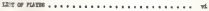

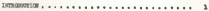

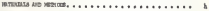

mons.

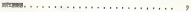

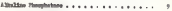

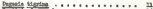

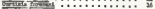

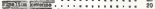

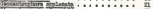

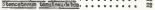

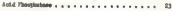

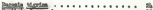

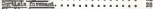

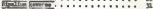

Oasontrefloge

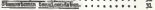

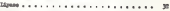

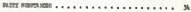

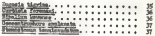


cus of eaveres igntised

$\operatorname{mos}$

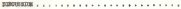

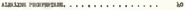

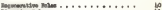

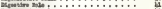

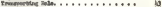

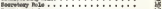

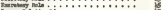

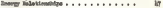

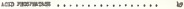

thate $, \ldots \ldots+\ldots, \ldots, \ldots, \ldots+\ldots$,

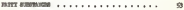

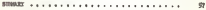

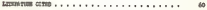

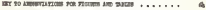

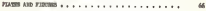

menama $\ldots, \ldots \ldots, \ldots, \ldots, \ldots, \ldots, \ldots$ n 


\section{Bex a vard}

7ove

her

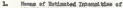

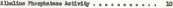

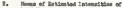

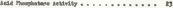




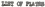

Moks

her

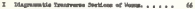

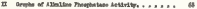

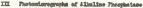

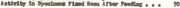

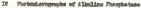

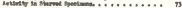

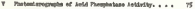

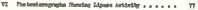

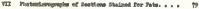

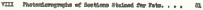




\section{Thionase}

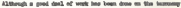

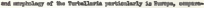

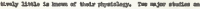

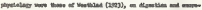

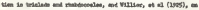

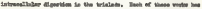

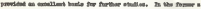

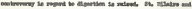

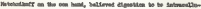

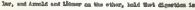

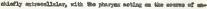

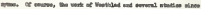

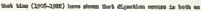

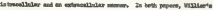

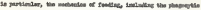

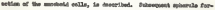

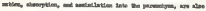
toustiod.

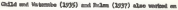

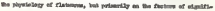
mats ta reosentim.

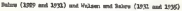

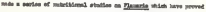
wodl is wormattin wis the poums wots. 


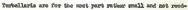

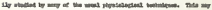

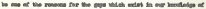

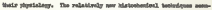

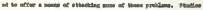

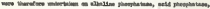

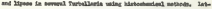

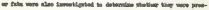

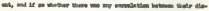

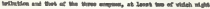
in eperted to ass ope thims,

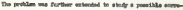

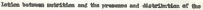

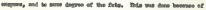

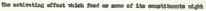

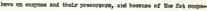

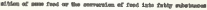

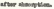

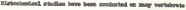

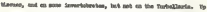

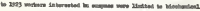

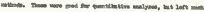

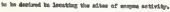

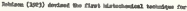

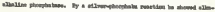

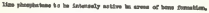




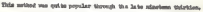

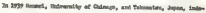

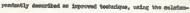

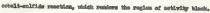

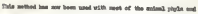
neve plethe

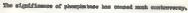

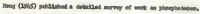

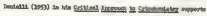

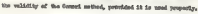

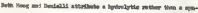

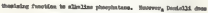

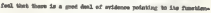

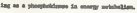

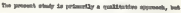

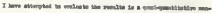

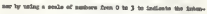

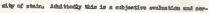

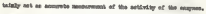

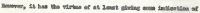

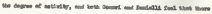

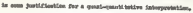




\section{monde dis urrom}

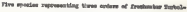

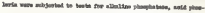

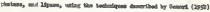

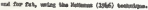

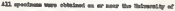

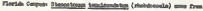

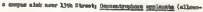

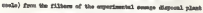

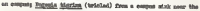

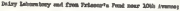

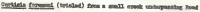

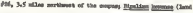

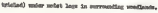

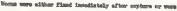

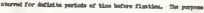

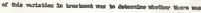

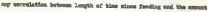
of elowes estinty.

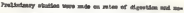

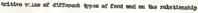

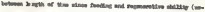

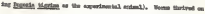

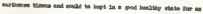

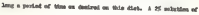




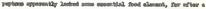

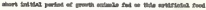

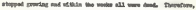

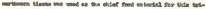

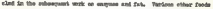

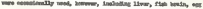
rali, and he liobs

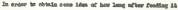

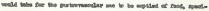

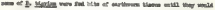

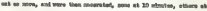

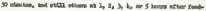

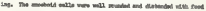

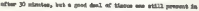

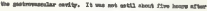

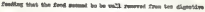
antor.

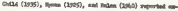

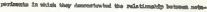

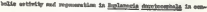

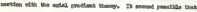

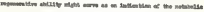

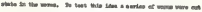

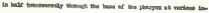

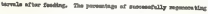

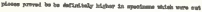




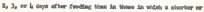

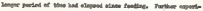

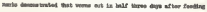

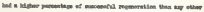

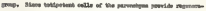

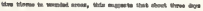

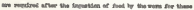

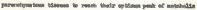

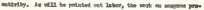

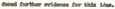

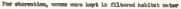

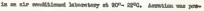

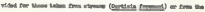

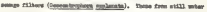

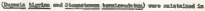

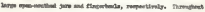

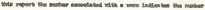

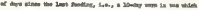

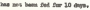

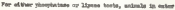

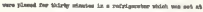

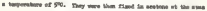

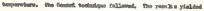

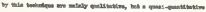

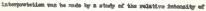




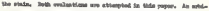

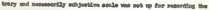

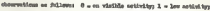

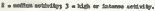

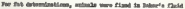

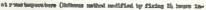

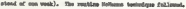

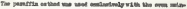

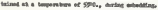

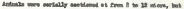

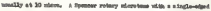
mave biolo mat.

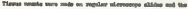

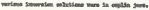

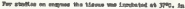

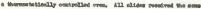

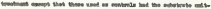

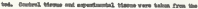

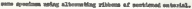

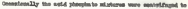

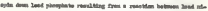

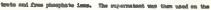
antrols intirs.

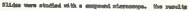

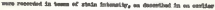


pasouryte.

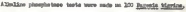

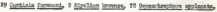

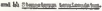

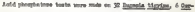

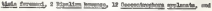

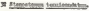

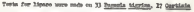

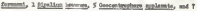

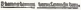

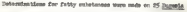

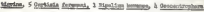

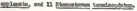

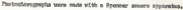




\section{$\operatorname{sen} 2$ \\ 1. therige \\ a. Hadion heratein}

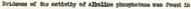

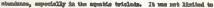

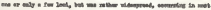

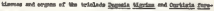

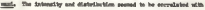

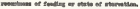

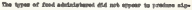

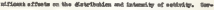

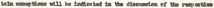

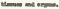

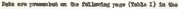

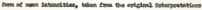

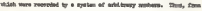

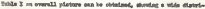

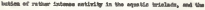

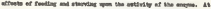

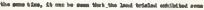

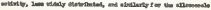

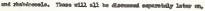

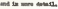




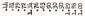

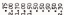

888

प⿺辶

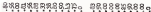

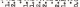

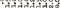

$111+1$

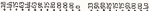

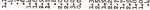

1:

$x^{2}$

Sita

能

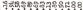

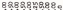

7.

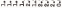

it

t $111+111111$

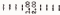

11

H1:H11111:

18 8R8

(4)

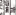

88

1 igg |

औ11

B I | | |

1191

111

te.

ted

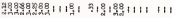

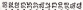

8.

1.2.

86

tit

a.s.

$\frac{7}{17}$

this

9.

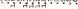

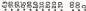
Ad

2.

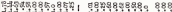
त.

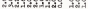

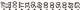
तลำ

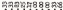

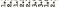

191

g. 8

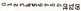

orinewembg oos
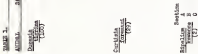

\& 8 . 2

늘

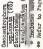




\section{Pranste 빔ote}

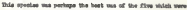

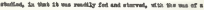

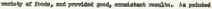

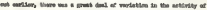

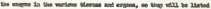

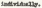

\section{thenen and orgint}

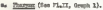

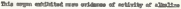

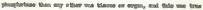

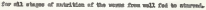

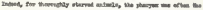

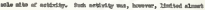

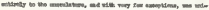

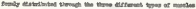

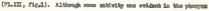

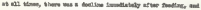

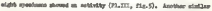

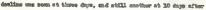

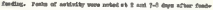

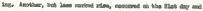

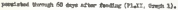




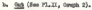

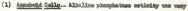

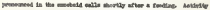

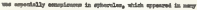

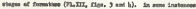

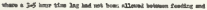

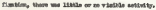

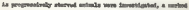

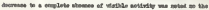

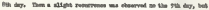

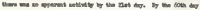

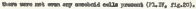

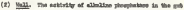

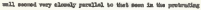

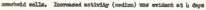

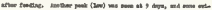

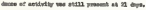

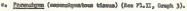

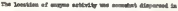

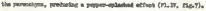

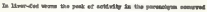

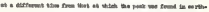

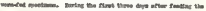

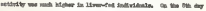

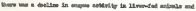




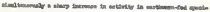
and

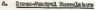

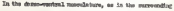

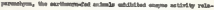

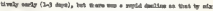

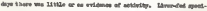

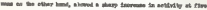
ders.

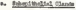

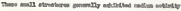

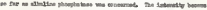

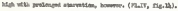

\section{Goesth}

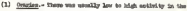

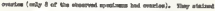

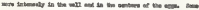

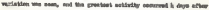
howding.

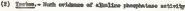

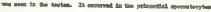

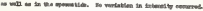

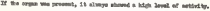




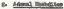

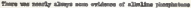

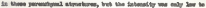

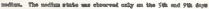
a chevelia indr, Misth.

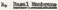

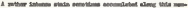

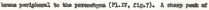

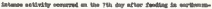

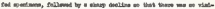

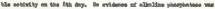

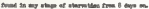

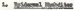

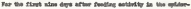

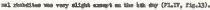

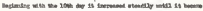

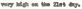

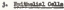

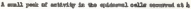

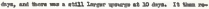

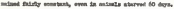

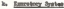

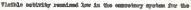




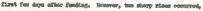

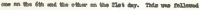

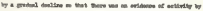

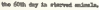

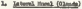

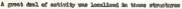

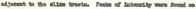

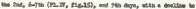

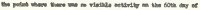
otanester.

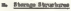

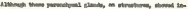

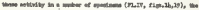

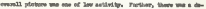

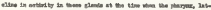

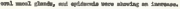

1. Exerested

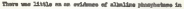

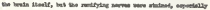

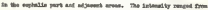

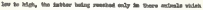
bal bes alemel har 60 dere. 


\section{Deftialn format}

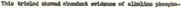

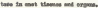

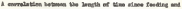

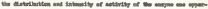

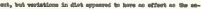

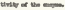

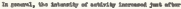

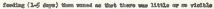

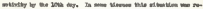

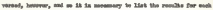
exas a blanse

\section{Finowe oul Drang}

\section{Roure}

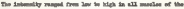

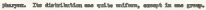

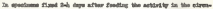

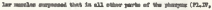

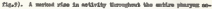

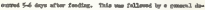

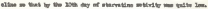

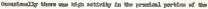

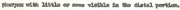

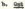

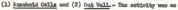




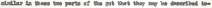

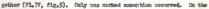

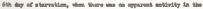

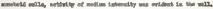

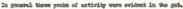

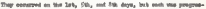

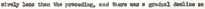

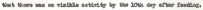

\section{c. Tysulpe}

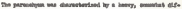

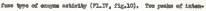

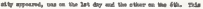

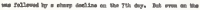

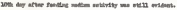

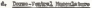

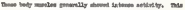

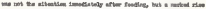

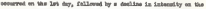

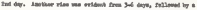

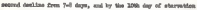

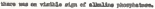

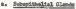

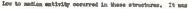

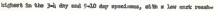

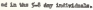




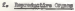

(1) ange

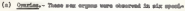

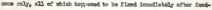

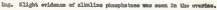

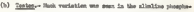

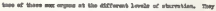

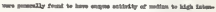

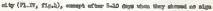
antirity.

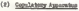

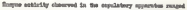

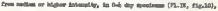

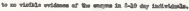

\section{(1) memendy}

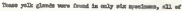

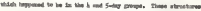

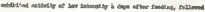

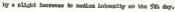

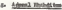

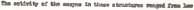

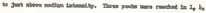

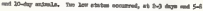
4an. 


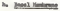

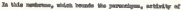

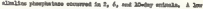

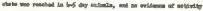

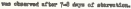

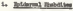

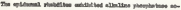

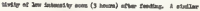

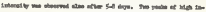

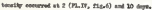

\section{Intparican}

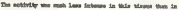

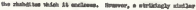

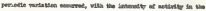

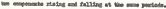

\section{Iiscian arte}

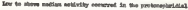

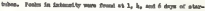

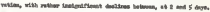

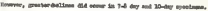

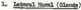

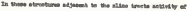

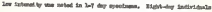




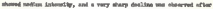

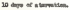

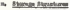

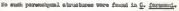

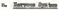

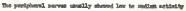

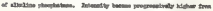

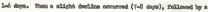

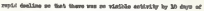
ntarvasion.

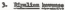

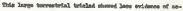

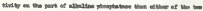

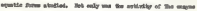

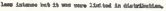

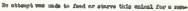

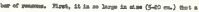

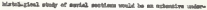

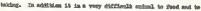

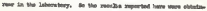

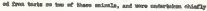

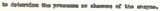

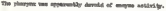

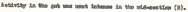




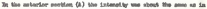

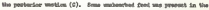

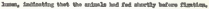

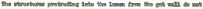

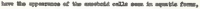

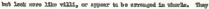

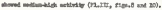

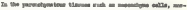

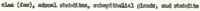

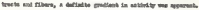

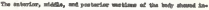

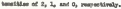

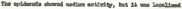

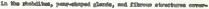

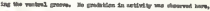

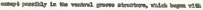

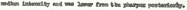

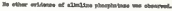

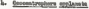

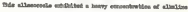

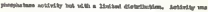

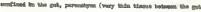

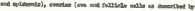

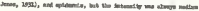

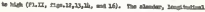




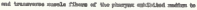

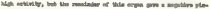

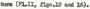

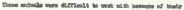

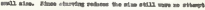

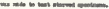

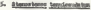

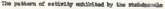

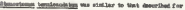

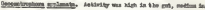

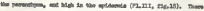

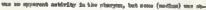

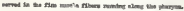

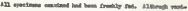

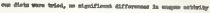
wars ipseanat. 


\section{A. Imst Manghange}

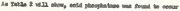

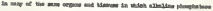

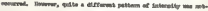

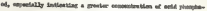

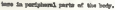

\section{pase 2}

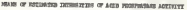

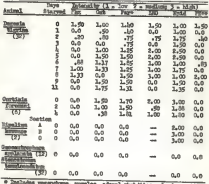

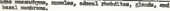

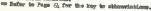




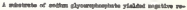

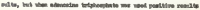

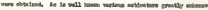

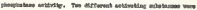

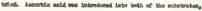

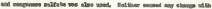

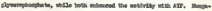

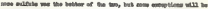
tegtelect.

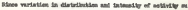

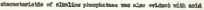

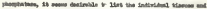
mines.

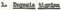

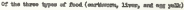

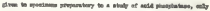

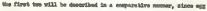

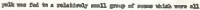

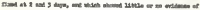
sell phanglatinge.

Therene and arat

a. Bunve

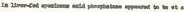

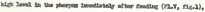




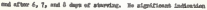

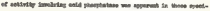

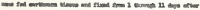
bucting:

i. 복

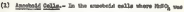

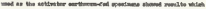

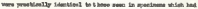

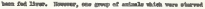

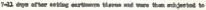

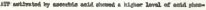

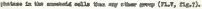

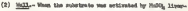

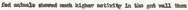

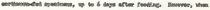

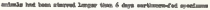

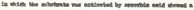

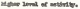

e. Nyoveres

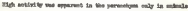

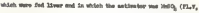

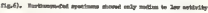

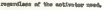




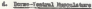

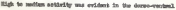

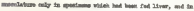

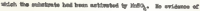

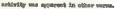

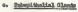

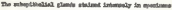

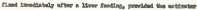

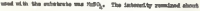

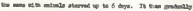

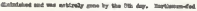

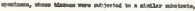

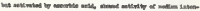

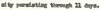

5. Oxan

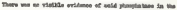
ands.

\section{1 ires Davation}

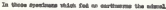

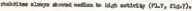

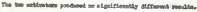

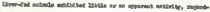

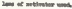




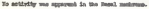

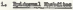

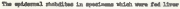

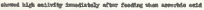

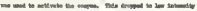

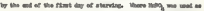

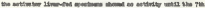

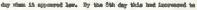

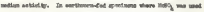

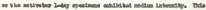

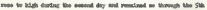

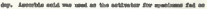

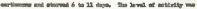

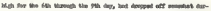

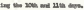

\section{Bothaser celle}

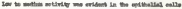

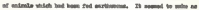

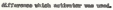

\section{b. Iminters grat}

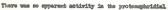
otratians. 


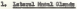

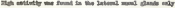

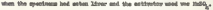

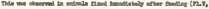

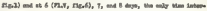

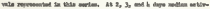

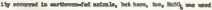

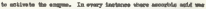

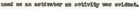

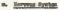

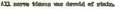

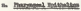

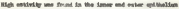

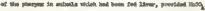

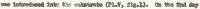

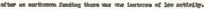

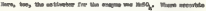

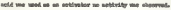

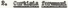

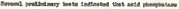

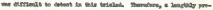

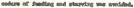

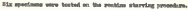




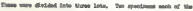

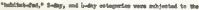

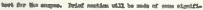

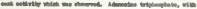

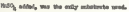

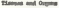

\section{Horne}

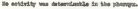

b. Den

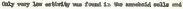

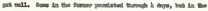

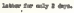

t. Varolega

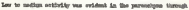
1 enth

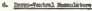

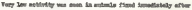

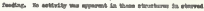
natoinal.

c. Bencibidet asong

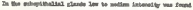

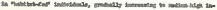

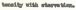




\section{Snople}

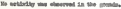

\section{Merer Reverese}

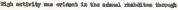

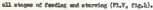

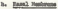

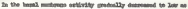

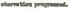

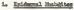

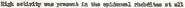

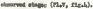

\section{S. Inthelt:?}

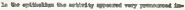

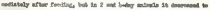
10u tabeusty.

\section{Ieretion trute.}

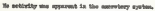

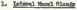

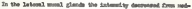

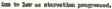

$=$ Danoves lat

13 nem thame me throse ef the shase, 


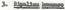

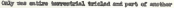

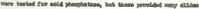

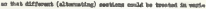

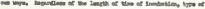

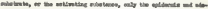

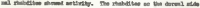

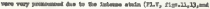

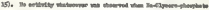

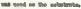

\section{Desentrerthen erciengle}

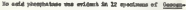

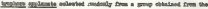

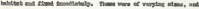

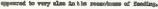

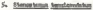

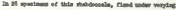

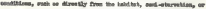

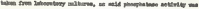
netbink 


\section{Uowe}

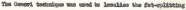

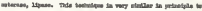

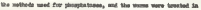

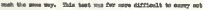

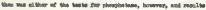
awe iave trotedeleath

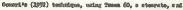

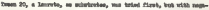

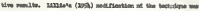

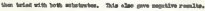

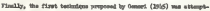

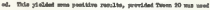
a to naturater.

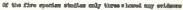

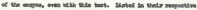

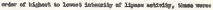

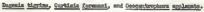

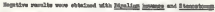
Lenderale

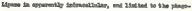

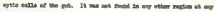

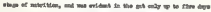

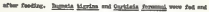

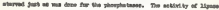




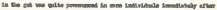

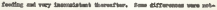

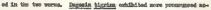

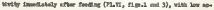

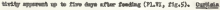

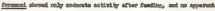

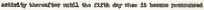

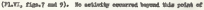
nurmation.

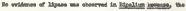

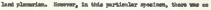

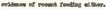

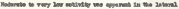

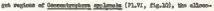

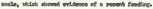

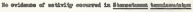

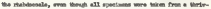

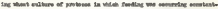
$27_{4}$ 


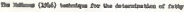

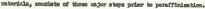

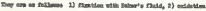

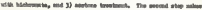

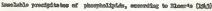

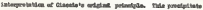

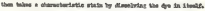

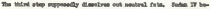

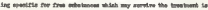

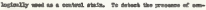

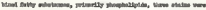

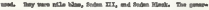

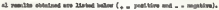

\section{Tuthy Bdablases}

\begin{tabular}{|c|c|c|c|c|}
\hline Istintran & 196h & Toxin & $\frac{2+a}{71}$ & 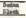 \\
\hline 모ave 2yos & 44 & 4 & $=$ & te \\
\hline Dupode 느gest & +4 & $*$ & $\bullet$ & qbo \\
\hline Goivole birint & & 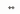 & $=$ & 4 \\
\hline 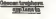 & 4 & + & + & 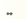 \\
\hline 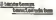 & $=$ & $=$ & = & + \\
\hline
\end{tabular}




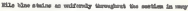

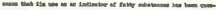

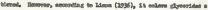

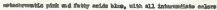

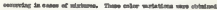

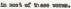

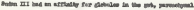

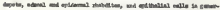

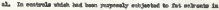

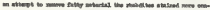

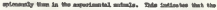

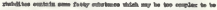

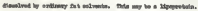

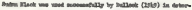

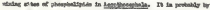

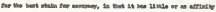
fer imoliods atowation.

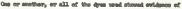

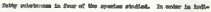

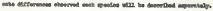

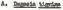

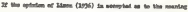

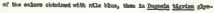

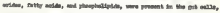

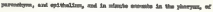




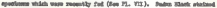

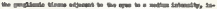

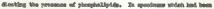

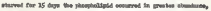

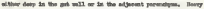

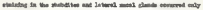

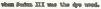

\section{Cirtate rerones}

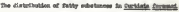

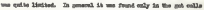

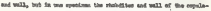

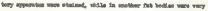

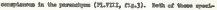

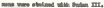

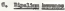

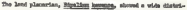

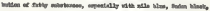

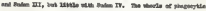

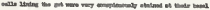

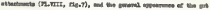

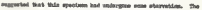

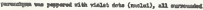

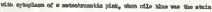

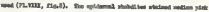




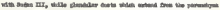

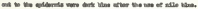

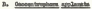

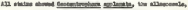

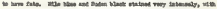

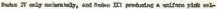

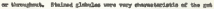

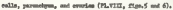

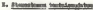

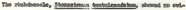

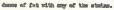




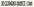

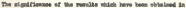

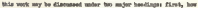

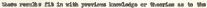

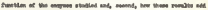

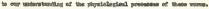

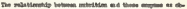

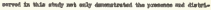

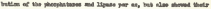

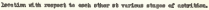

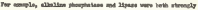

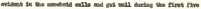

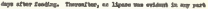

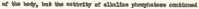

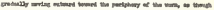

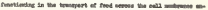

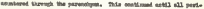

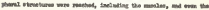

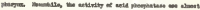

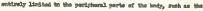

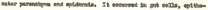

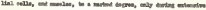
miturnatern.

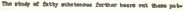

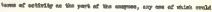




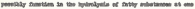

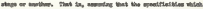

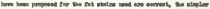

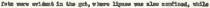

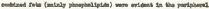

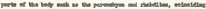

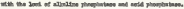

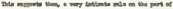

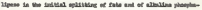

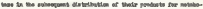

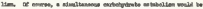

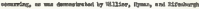

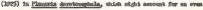

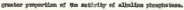

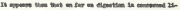

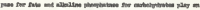

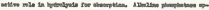

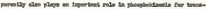

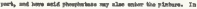

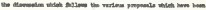

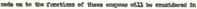

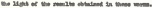




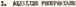

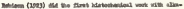

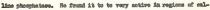

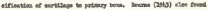

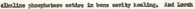

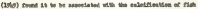

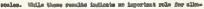

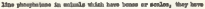

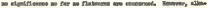

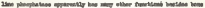
formater.

\section{Jecouentive miva}

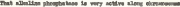

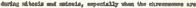

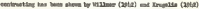

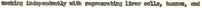

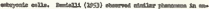

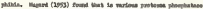

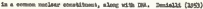

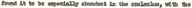

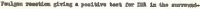

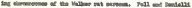

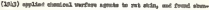

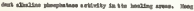

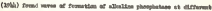




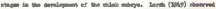

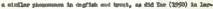
we at pouphats.

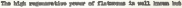

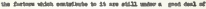

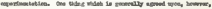

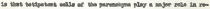

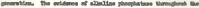

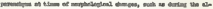

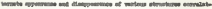

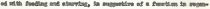

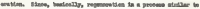

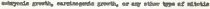

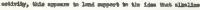

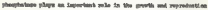
of extlat.

\section{B. Ditnenter thase}

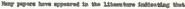

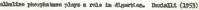

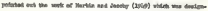

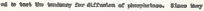

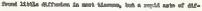

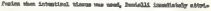

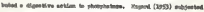

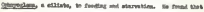




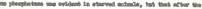

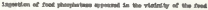

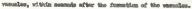

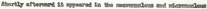

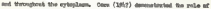

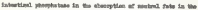

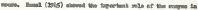

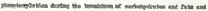

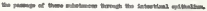

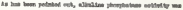

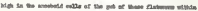

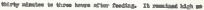

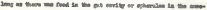

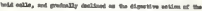

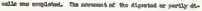

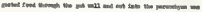

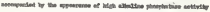

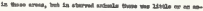

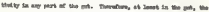

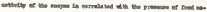

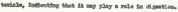

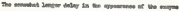

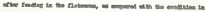

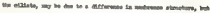

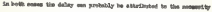




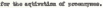

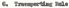

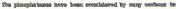

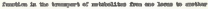

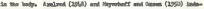

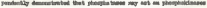

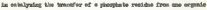

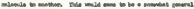

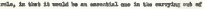

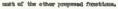

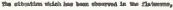

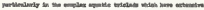

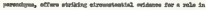

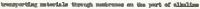

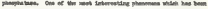

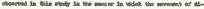

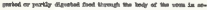

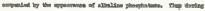

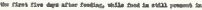

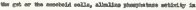

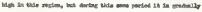

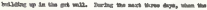

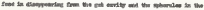

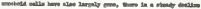

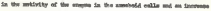




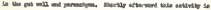

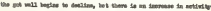

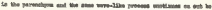

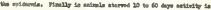

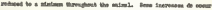

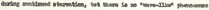

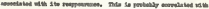

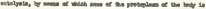

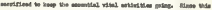

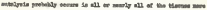

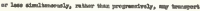

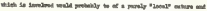

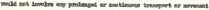

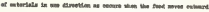

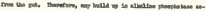

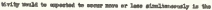

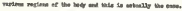

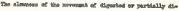

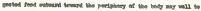

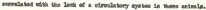

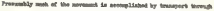

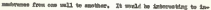

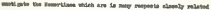

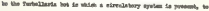

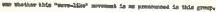




\section{2. tebotiong nala}

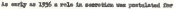

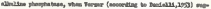

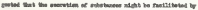

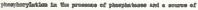

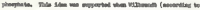

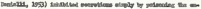

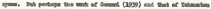

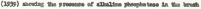

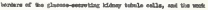

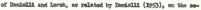

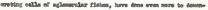

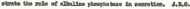

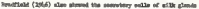

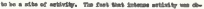

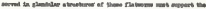

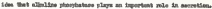

\section{Bunewry ind}

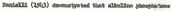

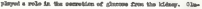

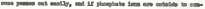

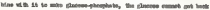

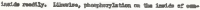

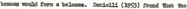




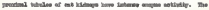

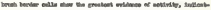

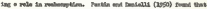

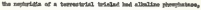

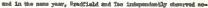

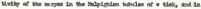

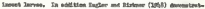

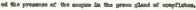

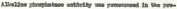

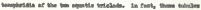

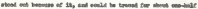

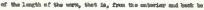

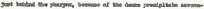

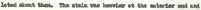

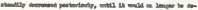
thetral.

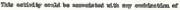

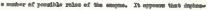

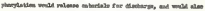

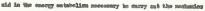

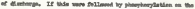

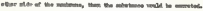

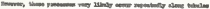

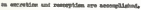




\section{Muro Jantamenge}

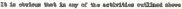

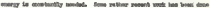

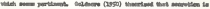

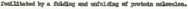

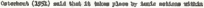

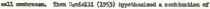

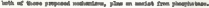

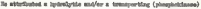

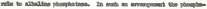

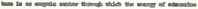

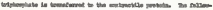

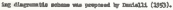

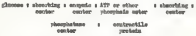

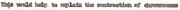

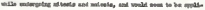

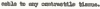

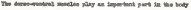

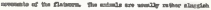

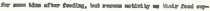

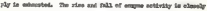

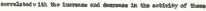




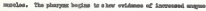

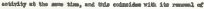

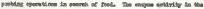

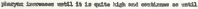

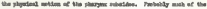

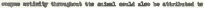

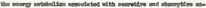

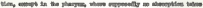
plomen, 


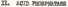

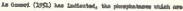

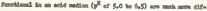

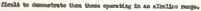

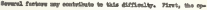

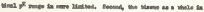

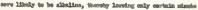

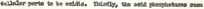

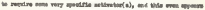

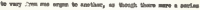

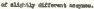

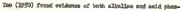

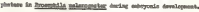

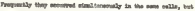

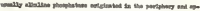

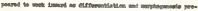

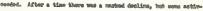

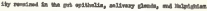

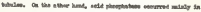

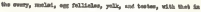

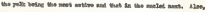

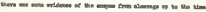

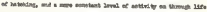

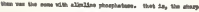

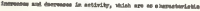




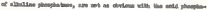
teneat

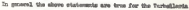

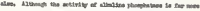

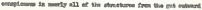

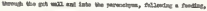

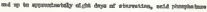

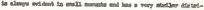

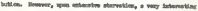

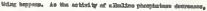

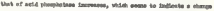

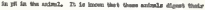

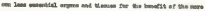

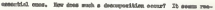

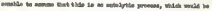

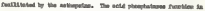

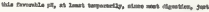

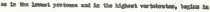

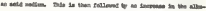

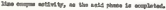

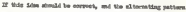

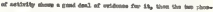

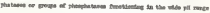

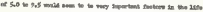

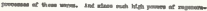

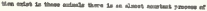




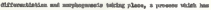

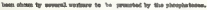

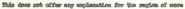

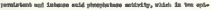

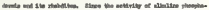

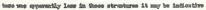

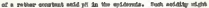

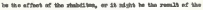
Find buabors af tam, 


\section{Iang}

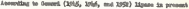

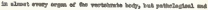

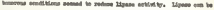

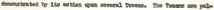

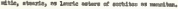

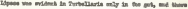

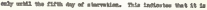

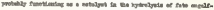

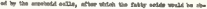

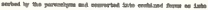

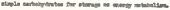

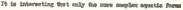

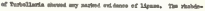

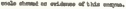

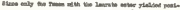

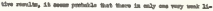

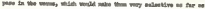
bye onowith. 


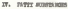

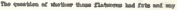

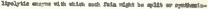

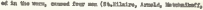

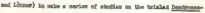

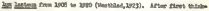

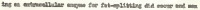

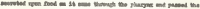

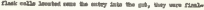

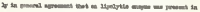

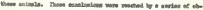

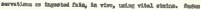

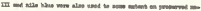

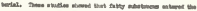

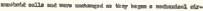

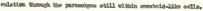

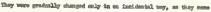

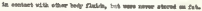

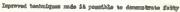

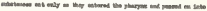

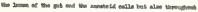

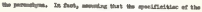

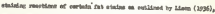

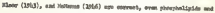

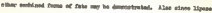




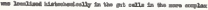

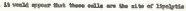

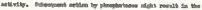

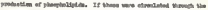

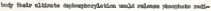

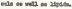

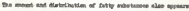

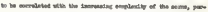

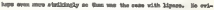

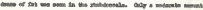

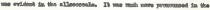

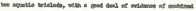

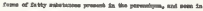

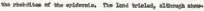

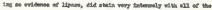

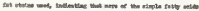

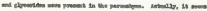

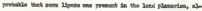

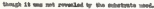

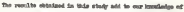

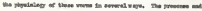

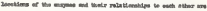

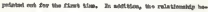

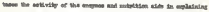

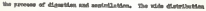




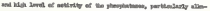

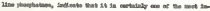

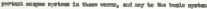

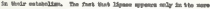

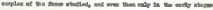

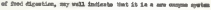

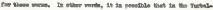

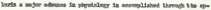

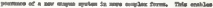

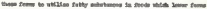

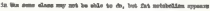

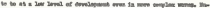

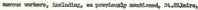

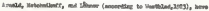

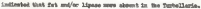

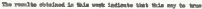

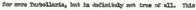

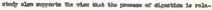

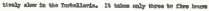

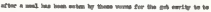

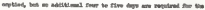

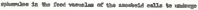

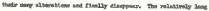

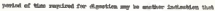

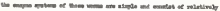


Itw avonim

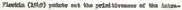

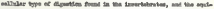

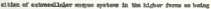

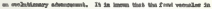

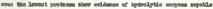

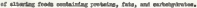

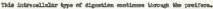

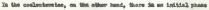

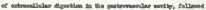

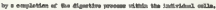

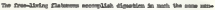

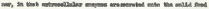

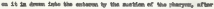

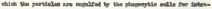

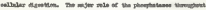

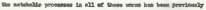

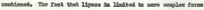

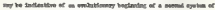

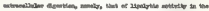
Inove

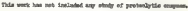

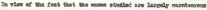

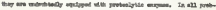

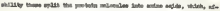

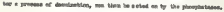




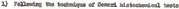

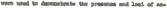

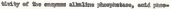

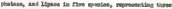

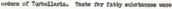

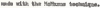

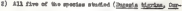

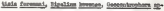

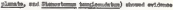

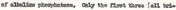

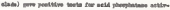

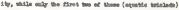

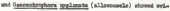
ones at Linow.

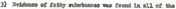

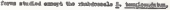

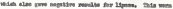

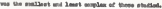

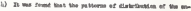

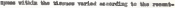

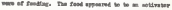

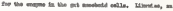




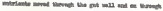

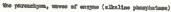

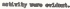

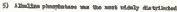

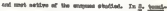

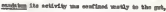

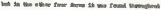
the bolp.

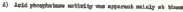

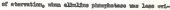

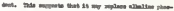

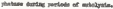

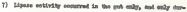

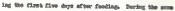

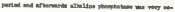

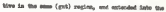
perwaten.

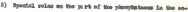

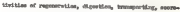

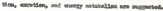

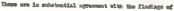

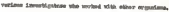

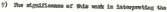




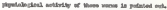

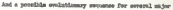

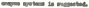




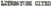

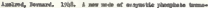

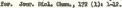

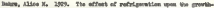

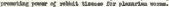

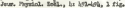

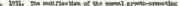

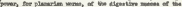

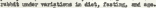

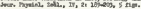

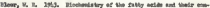

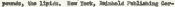

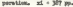

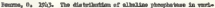

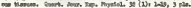

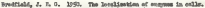

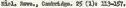

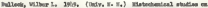

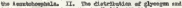

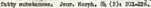

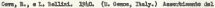

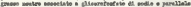

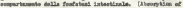

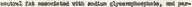

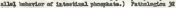
(ST)

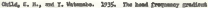

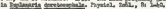

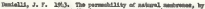

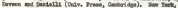

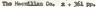

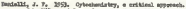

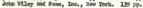

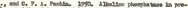

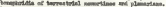

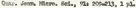




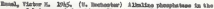

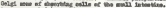

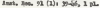

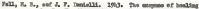

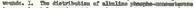

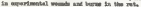

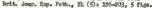

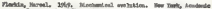
Prate. $1+159$ ine whill.

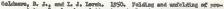

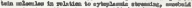

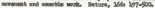

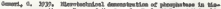

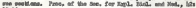
ijebr.

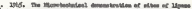

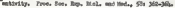

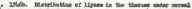

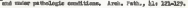

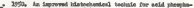

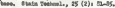

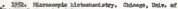

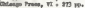

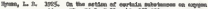

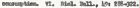

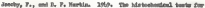

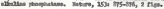

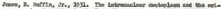

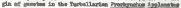

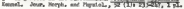

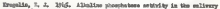

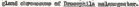

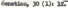

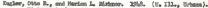

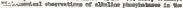

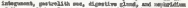

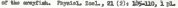




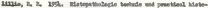

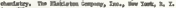
ix $=9 \mathrm{gat} \%$

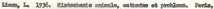

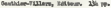

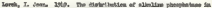

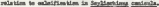

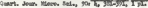

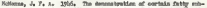

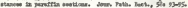

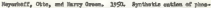

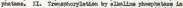

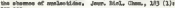
3rrolita

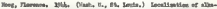

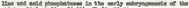

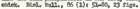

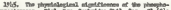

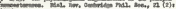
1A- 5 .

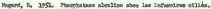

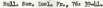

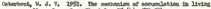

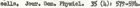

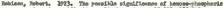

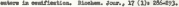

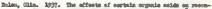

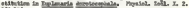
$10-1 \%$,

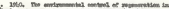

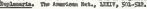

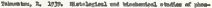

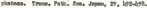

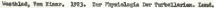

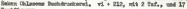
Frotifimon. 


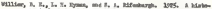

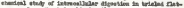

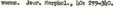

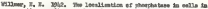

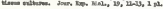

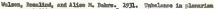

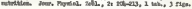

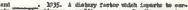

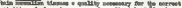

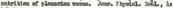

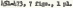

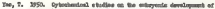

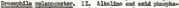

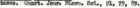




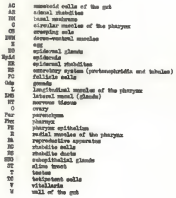




\section{retes $\mathbf{I}$}

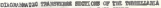

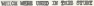

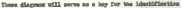

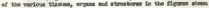

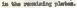

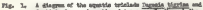

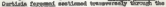

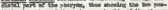

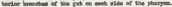

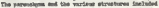

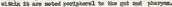

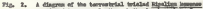

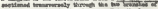

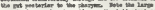

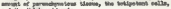
and the eifet epilaredis.

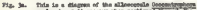

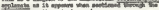

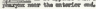

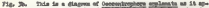

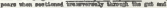

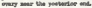

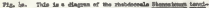

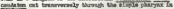

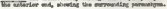

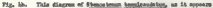

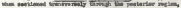

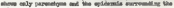
int. 


\section{purs:}

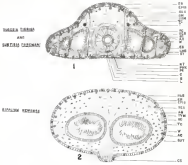

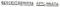
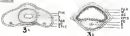

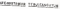
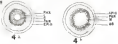


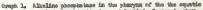

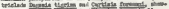

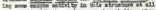

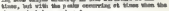

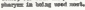

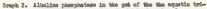

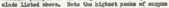

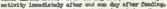

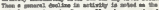

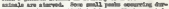

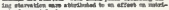

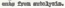

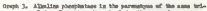

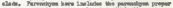

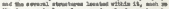

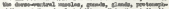

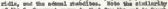
of we $\mathrm{g}$ frme

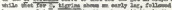

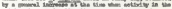

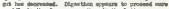

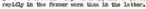




\section{Fer II}
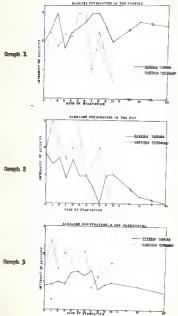

$-45=$ 


\section{Wra $\pi$}

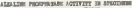

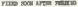

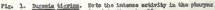

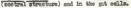

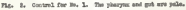

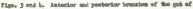

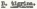

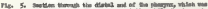

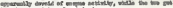

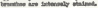

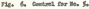

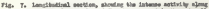

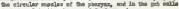

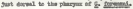

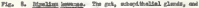

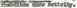

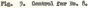

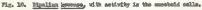

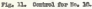

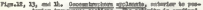

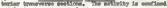

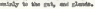

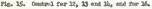

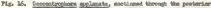

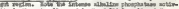

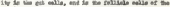
eviles.

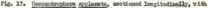

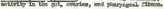

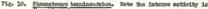

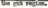

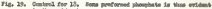
in the oulanter. 


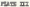
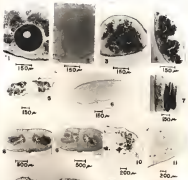

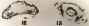

260

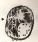

II
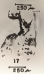

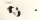

14
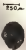

$\frac{10}{250-2}$ is 


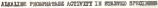

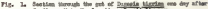

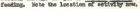

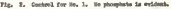

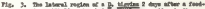

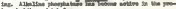

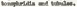

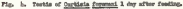

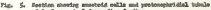

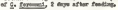

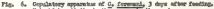

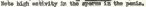

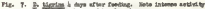

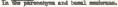

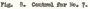

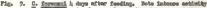

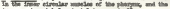

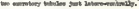

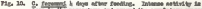

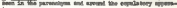
thas

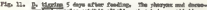

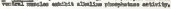

Fis. 12. Cercina thr log, 11 .

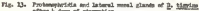

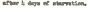

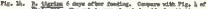

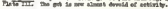

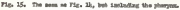


rua 17-Gedione

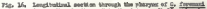

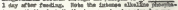

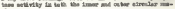
sinet.

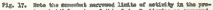

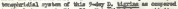

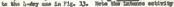

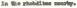

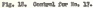

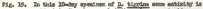

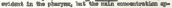

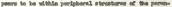

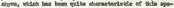

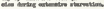

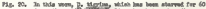

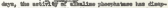

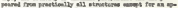

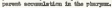


mast iv

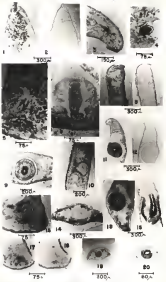




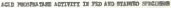

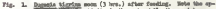

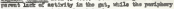

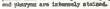

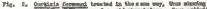

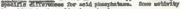

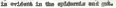

N5. 2. Gouknd Rot Bes 2.

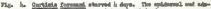

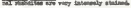

ng. 5. Cectave toe m, h.

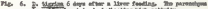

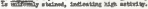

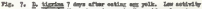

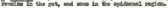

915. Ha Eeatrit the the 7.

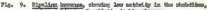

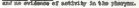

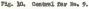

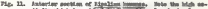

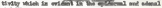
shabitstes

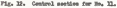

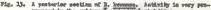

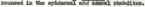

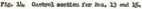

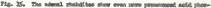

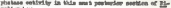
rehson lente. 


\section{nata}

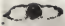

(5)
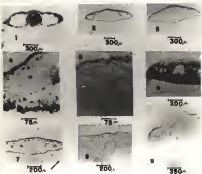

एकo.

300,

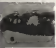

एकृष

TIE

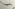

15 $+2+2$

?

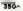

10
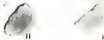

a.

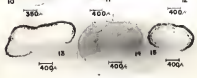




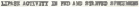

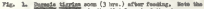

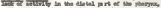

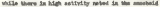

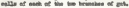

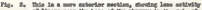

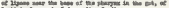

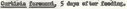

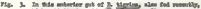

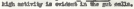

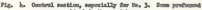

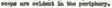

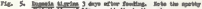

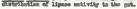

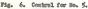

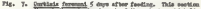

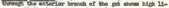

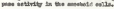

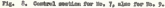

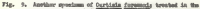

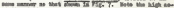

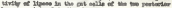
bencied, inberst the the phacgers

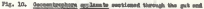

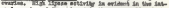

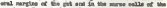

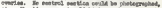

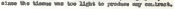



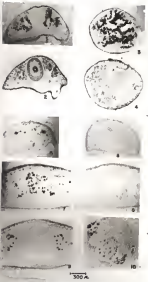


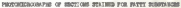

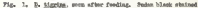

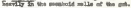

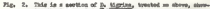

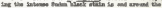

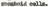

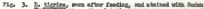

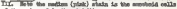

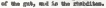

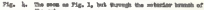
the erth

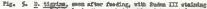

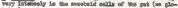

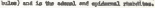

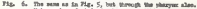

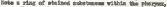

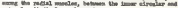

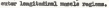

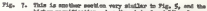

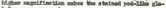
mins norv opewerk.

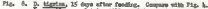

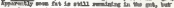

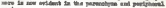
Tectsans,

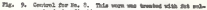

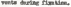

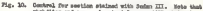

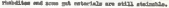

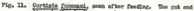

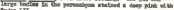
Bulae III.

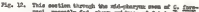

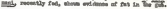


밤
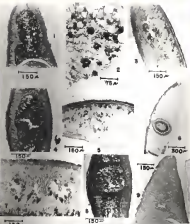

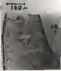

750
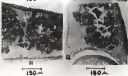


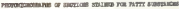

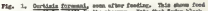

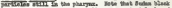

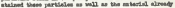

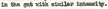

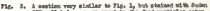

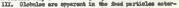

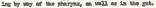

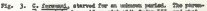
c.

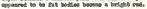

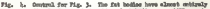

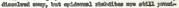
nowe

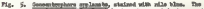

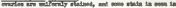

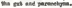

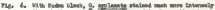

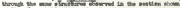

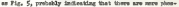

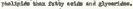

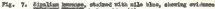

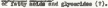

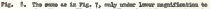

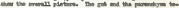

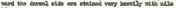
now. 
Nuse pot
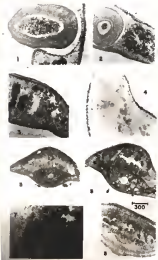

1505 


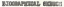

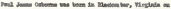

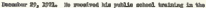

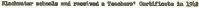

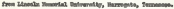

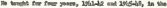

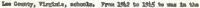

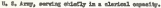

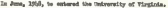

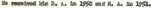

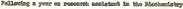

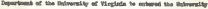

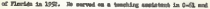

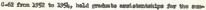

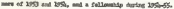

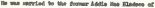

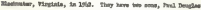

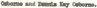

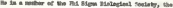

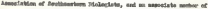
Ite Soetinty of the t10 It.

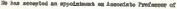

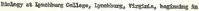

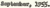




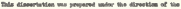

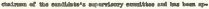

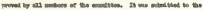

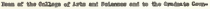

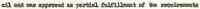

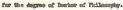

Iarses 13 , 1955

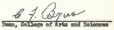

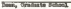

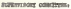
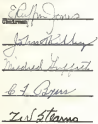